\title{
From femtonova to supernova: Heavy-ion collisions and the supernova equation of state
}

\author{
K. Hagel ${ }^{1}$, M. Hempel ${ }^{2}$ J. B. Natowitz ${ }^{1}$, G. Röpke ${ }^{3}$, S. Typel ${ }^{4}$, \\ S. Wuenschel ${ }^{1}$, R. WAdA $^{1}$, M. Barbui ${ }^{1}$ and K. Schmidt ${ }^{5}$
}

${ }^{1}$ Cyclotron Institute, Texas A \& M University, College Station, Texas, USA

${ }^{2}$ Department of Physics, University of Basel, 4056 Basel, Switzerland

${ }^{3}$ University of Rostock, FB Physik, Rostock, Germany

${ }^{4}$ GSI Helmholtzzentrum fr Schwerionenforschung GmbH, D-64291

Darmstadt, Germany

${ }^{5}$ Institute of Physics, Silesia University, Katowice, Poland

\begin{abstract}
Calculations using astrophysical equations of state at low densities comparable to that of the neutrino emission surface in supernovae and accretion disks are confronted with experimental results from heavy ion collisions. An extension of previous work shows that it is important to include all of the measured experimental data to draw conclusions about the astrophysical equation of state. Armed with this information, the calculations of the astrophysical equation of state are significantly constrained. Predictions of temperatures and densities sampled in black hole accretion disks are compared to those sampled in the experimental data.
\end{abstract}

\section{Introduction}

The evolution of explosions of massive stars samples densities from $10^{-10}<$ $\rho<2 \mathrm{nuc} / \mathrm{cm}^{3}$ and temperatures between $0<T<100 \mathrm{MeV}$. Ninety nine percent of the energy released in a core collapse supernova is radiated in 
neutrinos. The neutrinos interact until a temperature and density is reached where they no longer interact. This region is known as the neutrinosphere and is expected to have temperatures ranging from $5-10 \mathrm{MeV}$ and densities that range from $6 \times 10^{-5}-6 \times 10^{-3} \mathrm{fm}^{-3}$. Details of neutrino heating, therefore, depend on the properties of low density nuclear matter. Heavy ion collisions offer the possibility of studying low density nuclear matter and as such provide the intriguing possibility of studying stellar evolution and core-collapse supernovae $[1,2]$.

There are many calculations of core collapse supernovae [3-14] using different astrophysical equations of state (EOS). Calculations by design are performed with different assumptions and different compositions of competing particle species. In order to reduce discrepancies resulting from these different assumptions, we made comparisons of experimental data to the results of calculations using equilibrium constants [2]. The equilibrium constants are independent of particle species in the ideal gas limit of no interaction. When an interaction is present, differences in the equilibrium constants may provide a window into the nature of the interaction.

In reference [2], we compared $\alpha$-particle equilibrium constants from the experimental data to those from a number of supernovae calculations. The results showed that the experimental data significantly constrain the calculations. This work was extended to include all light particle species and more detail on the interaction, Coulomb effects and particle degrees of freedom [15]. These proceedings are, in part, a summary of that work.

The first extension of the analysis, in addition to that presented in [2], was to quantify the effect on the equilibrium constants extracted from $\alpha$ particles in [2] on the variation of different parameters in the same calculation. Figure 1 shows the results of varying the parameters of the calculation presented in [7]. The solid diamonds show the experimental data of [2] and the solid circles show the results of the ideal gas calculation whereas the open symbols show the equilibrium constants for the various parameters that are being varied. Panel (a) of figure 1 shows the dependence of the equilibrium constants on the particle composition assumed in the calculation. We note that the value of the equilibrium constants increases with increasing proton fraction, $y_{p}$.

Panel (b) of figure 1 shows a slight variation depending on whether Coulomb screening is present in the calculation or not. This is an important point in the comparison as supernova matter is charge neutral but the matter in heavy ion collisions has a net charge.

Finally panel (c) of figure 1 shows the variation with assumed particle degrees of freedom. The calculation shows almost no dependence when A is 


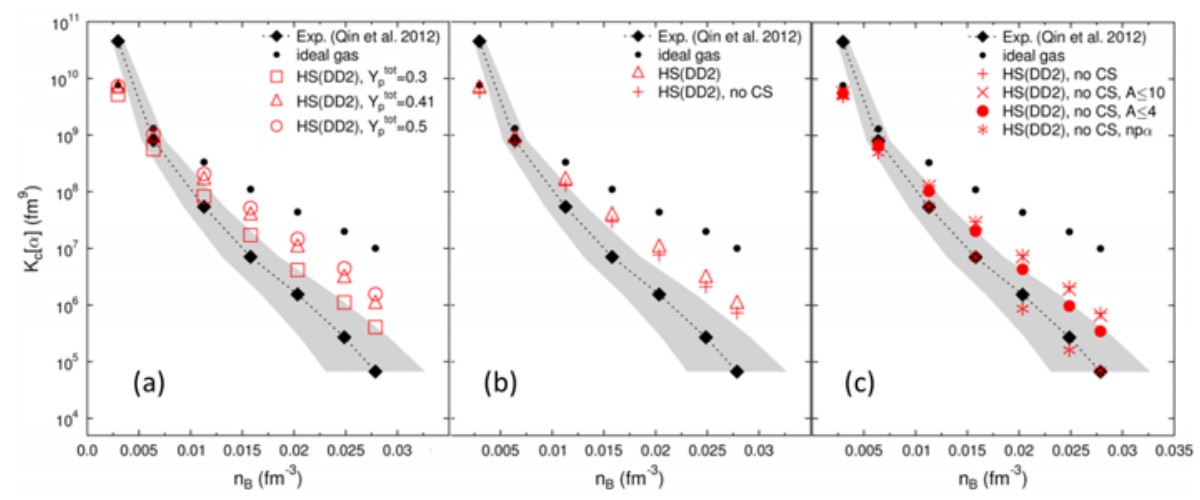

Figure 1: The dependence of $\alpha$-particle equilibrium constants on the variation of different parameters in the calculations. Panel (a) shows the variation with particle composition, panel (b) shows the variation with coulomb effects and panel (c) shows the variation with particle degrees of freedom.

constrained to be less than 10 as compared to no constraint. In the experiment there is, however, only a very small amount of fragment production of $\mathrm{A}>4$ from the source analyzed. The figure shows that there is a slight dependence when $\mathrm{A}$ is constrained to be less than 4 in the calculation.

The star symbols in panel (c) illustrate a very important point in comparing calculations of supernova EOSs to experimental data in general and heavy ion data in particular. Those equilibrium constants result from constraining the particle degrees of freedom to include only $\mathrm{n}, \mathrm{p}$ and $\alpha$. Taken by themselves, this implies the best agreement with the experimental data. This is, however, an accidental agreement as the experiment shows significant production of $\mathrm{d}, \mathrm{t},{ }^{3} \mathrm{He}$. The $\mathrm{d}, \mathrm{t},{ }^{3} \mathrm{He}$ equilibrium constants from calculations ignoring these species are by definition zero and that assumption will modify the results for the species included.

The rest of the calculations use the constraints that are present in the experiment. i. e., in the following $y_{p}=0.41$, Coulomb screening is turned off and the particle species are constrained to $\mathrm{A} \leq 4$ since those constraints are what was observed in the experiment.

Figure 2 shows the equilibrium constants with the experimental constraints for all of the light particles measured. As mentioned above, it is seen that while the calculation constraining production to $\mathrm{n}, \mathrm{p}$ and $\alpha$ agrees very well with the $\alpha$-particle equilibrium constants, this is partially because competing species are ignored. They cannot agree with the equilibrium constants of the other measured light particles. We do observe that the calculations that agree best with the experimental data are those where particle 

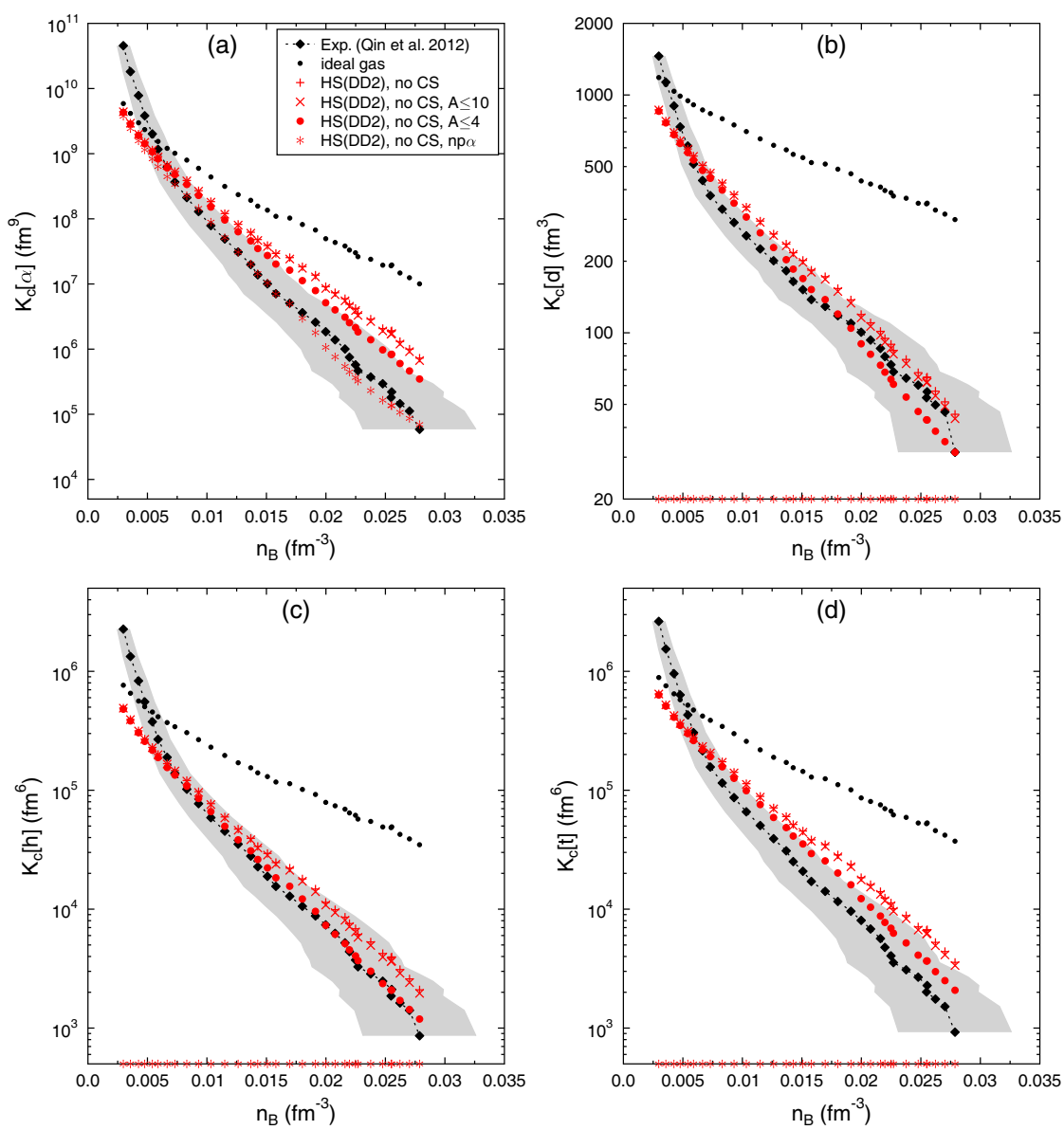

Figure 2: Equilibrium constants for light particles measured in the experiment. The experimental data is shown as solid diamonds, the ideal gas is shown as solid circles and the open symbols show the calculations with variations of different constraints on the particle degrees of freedom. Panel (a) shows the equilibrium constants for $\alpha$-particles, (b) for deuterons, (c) for tritons and (d) for ${ }^{3} \mathrm{He}$.

production is constrained to $\mathrm{A} \leq 4$.

As mentioned in the introduction, there are many calculations of the astrophysical EOS. Comparisons of results of other calculations to the experimental data have been made as well. Where possible the experimental constraints were added into the calculations. Figure 3 shows the results of these calculations. The figure shows two groups of calculations. The first group is the EOS calculations which include only n, p, $\alpha$ (plus a surrogate heavy species) and predict only $\mathrm{K}(\alpha)$, STOS [3,4], LS EOS [5], the 

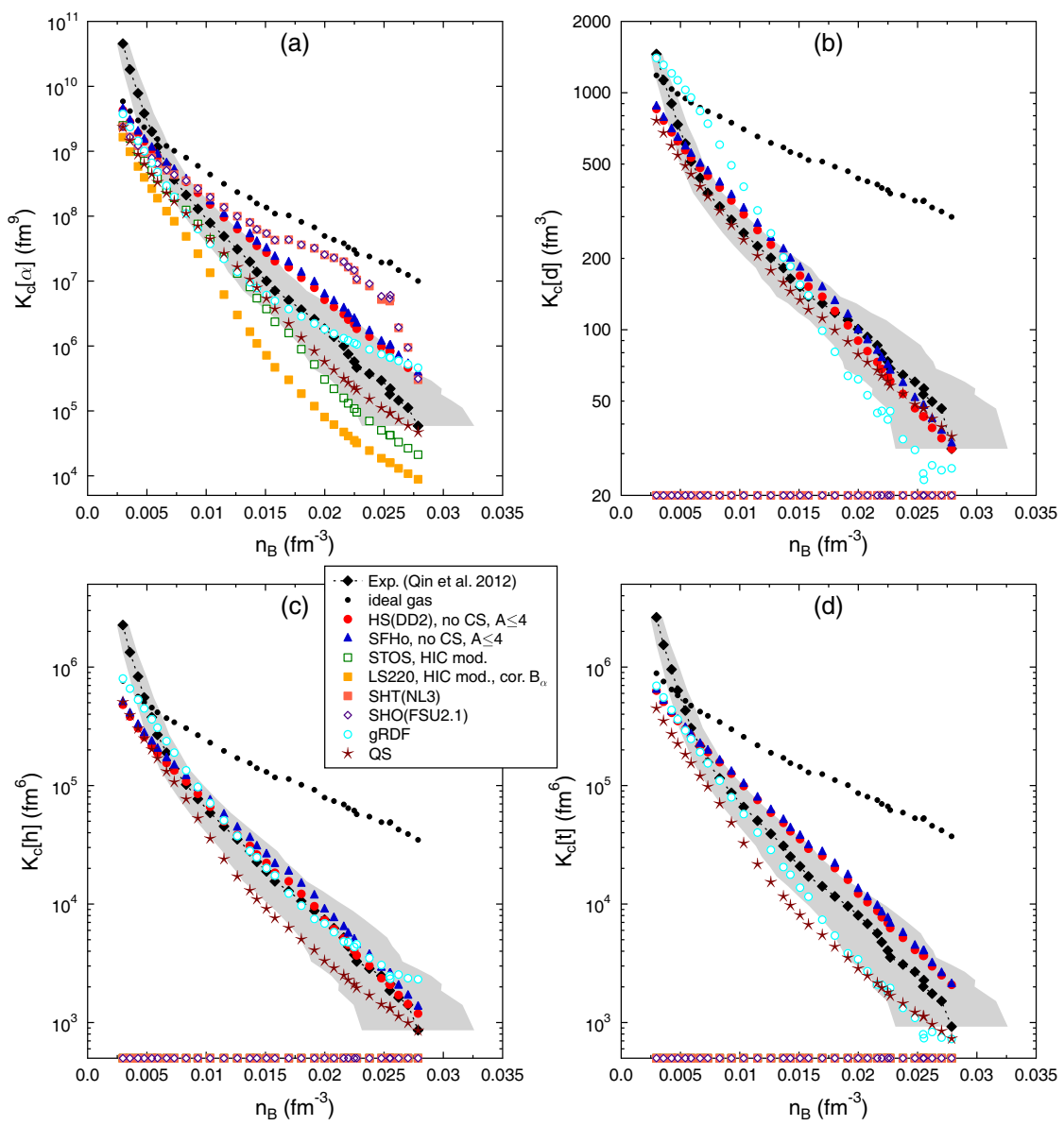

Figure 3: Equilibrium constants from experimental data compared to the predictions of various calculations of the astrophysical EOS. Calculations that treat only $\mathrm{n}, \mathrm{p}, \alpha$-particles are shown on the x-axis for particles other than $\alpha$-particles. See text for details.

NL3 [8] and FSU Gold [9]. The other models shown, HS(DD2) [7], SFHo [16], gRDF [11], and QS $[17,18]$ comprise a second group and these calculations include all of the particle species measured in the experiment. We observe that the equilibrium constants from all calculations converge to the ideal gas limit at low densities. The models that treat all light particles generally lie within the error bars of the experiment. The convergence at low densities is below the experimental data. This can probably be explained because the low energy particles leading to the equilibrium constants at low densities are emitted during the late stages of the reaction where there is probably con- 


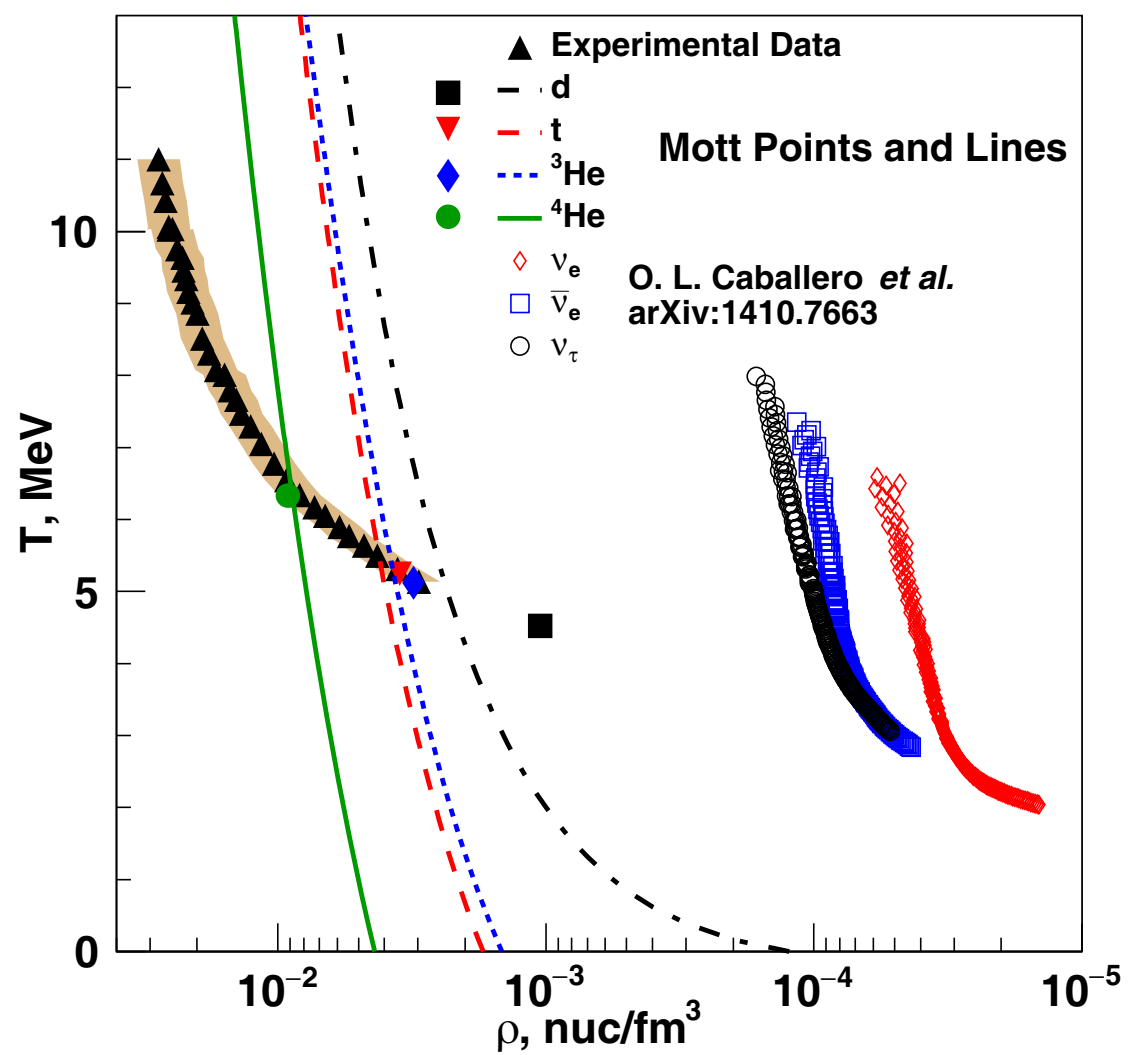

Figure 4: The evolution of $\mathrm{T}$ vs $\rho$. Solid triangles indicate the experimental data. Solid squares, inverted triangles, diamonds and circles indicate Mott points shown in [20]. Open diamonds, squares and circles indicate the temperature density map of neutrinos from black hole accretion disks

tamination by particles from sources other than the coalescing low density gas. Such contributions of low energy particles are very difficult to separate.

We have also explored how the density vs temperature profiles sampled in the neutrinosphere or in accretion disks compares to the density vs temperature profile that is sampled in our experimental data. The solid triangles in figure 4 show the density vs temperature profile from our experimental data $[2,19,20]$. Note the reversed axis in density. The other solid symbols show the Mott points extracted from the experimental data [20] along with theoretical predictions of the temperature vs density profile of the Mott 
points [7]. The open diamonds, squares and circles show the prediction of the temperature vs density sampling from black hole accretion disks $[21,22]$ for neutrinos, anti-neutrinos, and tau-neutrinos, respectively.

We observe from the figure that heavy ion collisions do indeed sample temperatures and densities in the vicinity of the neutrinosphere. The calculations from black hole accretion disks indicate that similar temperatures are sampled, but that the density sampled in the experiment is higher.

In summary we have significantly constrained calculations of the astrophysical EOS. An extension of the work in [2] has shown that it is important to include all of the measured experimental data in order to draw meaningful conclusions [15] on the astrophysical EOS. We have also compared the evolution of temperature and density that is sampled in heavy in collisions to the predictions of that sampled by neutrinos in black hole accretion disks. The experiment is shown to be sampling nuclear matter in the range of the neutriosphere and black hole accretion disks.

This work was supported by the United States Department of Energy under Grant \# DE-FG03- 93ER40773 and by The Robert A. Welch Foundation under Grant \# A0330.

\section{References}

[1] B. A. Li, L. W. Chen and C. M. Ko, Phys. Rep. 464, 113 (2008).

[2] L. Qin et al., Phys. Rev. Lett. 108, 172701 (2012).

[3] H. Shen et al., Nucl. Phys. A637, 435 (1998); Prog. Theor. Phys. 100, 1013 (1998).

[4] H. Shen, H. Toki, K. Oyamatsu, and K. Sumiyoshi, Astrophys. J. Suppl. 197, 20 (2011).

[5] J. M. Lattimer and F. D. Swesty, Nucl. Phys. A535, 331 (1991).

[6] C. J. Horowitz and A. Schwenk, Nucl. Phys. A776, 55 (2006).

[7] S. Typel, G. Röpke, T. Klahn, D. Blaschke and H. H. Wolter, Phys. Rev. C 81, 015803 (2010).

[8] G. Shen, et al., Phys. Rev. C 83, 035802 (2011).

[9] G. Shen et al., Phys. Rev. C 83, 065808 (2011).

[10] M. Hempel and J. Schaffner-Bielich, Nucl. Phys. A837, 210 (2010). 
[11] M. D. Voskresenskaya and S. Typel, Nucl. Phys. A887, 42 (2012).

[12] G. Röpke, M. Schmidt, L.Münchow, and H. Schulz, Nucl. Phys. A399, 587 (1983).

[13] G. Röpke, L. Münchow, and H. Schulz, Nucl. Phys. A379, 536 (1982).

[14] G. Röpke, L. Münchow, and H. Schulz, Phys. Lett. B110, 21 (1982).

[15] M. Hempel et al., Phys. Rev. C 91, 045805 (2015).

[16] A. W. Steiner, M. Hempel, and T. Fischer, Astrophys. J. 774, 17 (2013). (2013).

[17] G. Röpke, Phys. Rev. C 79, 014002 (2009).

[18] G. Röpke, Nucl. Phys. A 867, 66 (2011).

[19] R. Wada et al., Phys. Rev. C 85, 064618 (2012).

[20] K. Hagel et al., Phys. Rev. Lett. 108, 062702 (2012).

[21] O. L. Caballero et al., ArXiv:1410.7663 (2014).

[22] O. L. Caballero et al., EPJ Web of Conferences 93, 03002 (2015). 